2

3

4

\title{
Bevacizumab treatment of patients with breast cancer is associated with induction of SOX17.
}

Shahan Mamoor, MS 1

1shahanmamoor@gmail.com

East Islip, NY USA

Bevacizumab (Avastin) is an approved treatment option by the European Medicines Agency ${ }^{1}$ for more than a quarter billion women in the European Union, and despite having its indication withdrawn by the Food and Drug Administration in 2011 is still utilized in clinical trials in the United States ${ }^{2,3}$. We mined published microarray data ${ }^{4}$ from the PROMIX trial to understand in an unbiased fashion genes most transcriptionally perturbed by bevacizumab administration and how this interacted with a standard anthracycline and taxane chemotherapeutic regimen, epirubicin and docetaxel. We report here the striking induction of the SRY-box morphogen transcription factor SOX175-7 in the primary tumors of women with breast cancer treated with bevacizumab.

Keywords: breast cancer, SOX17, bevacizumab, Avastin, transcription factors in breast cancer, systems biology of breast cancer, targeted therapeutics in breast cancer. 
Breast cancer affects women worldwide with relatively high frequency ${ }^{8}$. Bevacizumab (Avastin), a monoclonal antibody targeted against the vascular endothelial growth factor A, VEGF-A, is utilized for the treatment of breast cancer worldwide9. Bevacizumab was formerly approved by the Food and Drug Administration in conjunction with paclitaxel for the treatment of metastatic breast cancer which had not previously been treated with chemotherapy for HER2-negative breast cancer; this indication was withdrawn on the basis that its full safety and efficacy had not been demonstrated ${ }^{2}$. For the over quarter of a billion women in the European Union, bevacizumab remains approved by the European Medicines Agency for the treatment of metastatic breast cancer ${ }^{1}$. To understand the transcriptional response to bevacizumab in an unbiased fashion at the transcriptome level, we mined published microarray data from $\mathrm{PROMIX}^{4}$, a phase II clinical trial measuring clinical responses to epirubicin, docetaxel and bevacizumab in women with breast cancer.

\section{Methods}

\section{Results}

We mined published microarray data 4,10 to understand in an unbiased fashion and at the systems level how transcription globally was most significantly altered in primary tumors from humans with cancer following treatment with the monoclonal antibody bevacizumab.

\section{Bevacizumab treatment results in transcriptional induction of SOX17 in the primary tumors of women with breast cancer.}

We analyzed data from the PROMIX trial ${ }^{4}$ wherein patients were treated for 6 cycles; each of the six cycles included treatment with epirubicin and docetaxel; bevacizumab was added to the treatment regimen only after cycle 2 . Tumor tissues were sampled at baseline, following administration of 2 cycles 
of epirubicin and docetaxel (prior to administration of bevacizumab), and after the addition of bevacizumab, at surgery. We identified the SRY-box 17 transcription factor SOX17 as among the genes most differentially expressed in the primary tumors of human patients with breast cancer when comparing tumors at baseline, after 2 cycles of epirubicin and docetaxel, and at surgery, once bevacizumab had been administered for 4 cycles (Table 1). When sorting each of the genes expressed in the primary tumors of humans with breast cancer as compared to baseline, after 2 cycles of epirubicin and docetaxel, and after 4 cycles of bevacizumab, epirubicin and docetaxel, for 6 cycles of total treatment, SOX17 ranked 164 out of 47323 total transcripts (Table 1). Differential expression of SOX17 (Table 1; $p=1.04 \mathrm{E}-15$ ) in the primary tumors of women with breast cancer following treatment with bevacizumab, epirubicin and docetaxel was statistically significant.

Next we evaluated the magnitude and direction of change in SOX17 gene expression in the primary tumors of breast cancer patients after 2 cycles of epirubicin and docetaxel as well as once bevacizumab had been added to the treatment regimen for 4 cycles. Remarkably, we found that each of these transcription factors significantly increased in expression, but only after treatment with baseline). We calculated a mean fold change of $1.26 \pm 0.35$ in SOX17 gene expression following addition of bevacizumab to the treatment regimen (Table 1).

Bevacizumab treatment results in transcriptional induction of SOX17 in the tumors of patients with colorectal cancer.

We turned to a second microarray dataset ${ }^{10}$ to understand more thoroughly tissue-specific transcriptional modulation by bevacizumab in cancer, comparing global gene expression between the tumors of patients treated with FOLFIRI chemotherapeutic regimen or the tumors of patients treated with FOLFIRI and bevacizumab. We found SOX17 was among the genes most differentially expressed in the tumors of patients with colorectal cancer upon treatment with bevacizumab (Table 2). When sorting each of the genes expressed in primary colorectal tumors based on significance of change in expression 54765 total transcripts (Table 2). Differential expression of SOX17 in the primary tumors of bevacizumab-treated patients with colorectal cancer was statistically significant (Table $2 ; \mathrm{p}=2.68 \mathrm{E}-05$ ).

We also assessed magnitude and direction of SOX17 transcriptional modulation by bevacizumab primary colorectal tumors of patients treated with bevacizumab and FOLFIRI as compared to patients treated with FOLFIRI only (Figure 2), just as we did in patients with breast cancer. We calculated a mean fold change of $1.10 \pm 0.13$ in SOX17 gene expression in the colorectal tumors of patients treated with bevacizumab and FOLFIRI as compared to primary tumors from patients treated with FOLFIRI only. Transcriptional induction of SOX17 in the primary tumors of mice treated with bevacizumab was statistically significant (Figure $2 ; \mathrm{p}=0.0021$ ).

\section{Discussion}

Among the described functions of SOX17 are the specification of primordial germ cell fate 5 , specification and maintenance of arterial tissue identity ${ }^{6}$, and in the regulation of oligodendrocyte cell fate7. We provided evidence here that an SRY-box morphogen transcription factor is among the genes whose expression changes most significantly in primary tumors of the breast and of the colon and rectum upon treatment with bevacizumab. Thus, a treatment whose direct molecular action is inhibition of a vascular endothelial growth factor results in transcriptional induction of a morphogen transcription factor required for specification of arterial identity ${ }^{6}$. 


\section{References}

1. Avastin. European Medicines Agency. https://www.ema.europa.eu/en/medicines/human/EPAR/ avastin.

2. Lenzer, J., 2011. FDA committee votes to withdraw bevacizumab for breast cancer.

3. Basho, R.K., Gilcrease, M., Murthy, R.K., Helgason, T., Karp, D.D., Meric-Bernstam, F., Hess, K.R., Herbrich, S.M., Valero, V., Albarracin, C. and Litton, J.K., 2017. Targeting the PI3K/AKT/mTOR pathway for the treatment of mesenchymal triple-negative breast cancer: evidence from a phase 1 trial of mTOR inhibition in combination with liposomal doxorubicin and bevacizumab. JAMA oncology, 3(4), pp.509-515.

4. Kimbung, Siker, Ida Markholm, Judith Bjöhle, Tobias Lekberg, Anna Von Wachenfeldt, Edward Azavedo, Ariel Saracco et al. "Assessment of early response biomarkers in relation to long-term survival in patients with HER2-negative breast cancer receiving neoadjuvant chemotherapy plus bevacizumab: results from the Phase II PROMIX trial." International journal of cancer 142, no. 3 (2018): 618-628.

5. Irie, N., Weinberger, L., Tang, W.W., Kobayashi, T., Viukov, S., Manor, Y.S., Dietmann, S., Hanna, J.H. and Surani, M.A., 2015. SOX17 is a critical specifier of human primordial germ cell fate. Cell, 160(1-2), pp.253-268.

6. Corada, M., Orsenigo, F., Morini, M.F., Pitulescu, M.E., Bhat, G., Nyqvist, D., Breviario, F., Conti, V., Briot, A., Iruela-Arispe, M.L. and Adams, R.H., 2013. Sox17 is indispensable for acquisition and maintenance of arterial identity. Nature communications, 4, p.2609.

7. Sohn, J., Natale, J., Chew, L.J., Belachew, S., Cheng, Y., Aguirre, A., Lytle, J., Nait-Oumesmar, B., Kerninon, C., Kanai-Azuma, M. and Kanai, Y., 2006. Identification of Sox17 as a transcription factor that regulates oligodendrocyte development. Journal of Neuroscience, 26(38), pp.9722-9735.

8. Bray, F., Ferlay, J., Soerjomataram, I., Siegel, R.L., Torre, L.A. and Jemal, A., 2018. Global cancer statistics 2018: GLOBOCAN estimates of incidence and mortality worldwide for 36 cancers in 185 countries. CA: a cancer journal for clinicians, 68(6), pp.394-424.

9. Ellis, L.M. and Kirkpatrick, P., 2005. Bevacizumab. Nature Reviews Drug Discovery, 4.

10.Del Rio, M., Mollevi, C., Bibeau, F., Vie, N., Selves, J., Emile, J.F., Roger, P., Gongora, C., Robert, J., Tubiana-Mathieu, N. and Ychou, M., 2017. Molecular subtypes of metastatic colorectal cancer are associated with patient response to irinotecan-based therapies. European Journal of Cancer, 76, pp. $68-75$.

PAGE 4 
PAGE 5 


\begin{tabular}{|c|c|c|c|c|c|c|c|}
\hline Rank & ID & $p$-value & $\mathbf{t}$ & B & FC & gene & Gene name \\
\hline 161 & 230943_at & 2.68E-05 & 4.4468534 & 2.37954 & $1.10 \pm 0.13$ & SOX17 & SRY-box 17 \\
\hline
\end{tabular}

Table 2: Differential expression of SOX17 in colorectal tumors of patients treated with bevacizumab.

The rank of differential expression, probe ID, $p$-value with respect to global differential expression, $t$, a moderated t-statistic, B, the log-odds of differential expression between the two groups compared, fold change in SOX17 gene expression between tumors of patients treated with bevacizumab and FOLFIRI as compared to that of patients treated with FOLFIRI only, gene and gene name are listed in this chart. 


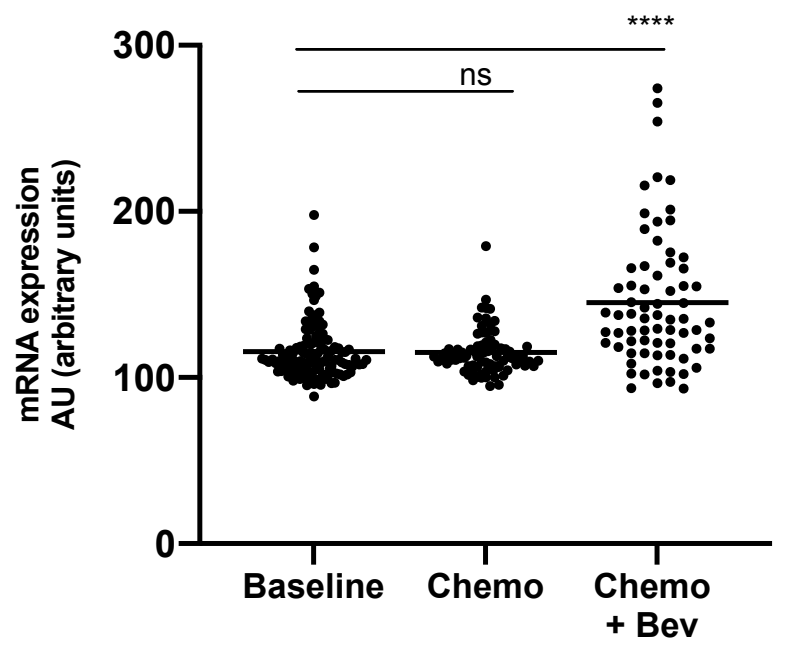

Figure 1: SOX17 is transcriptionally induced in the breast tumors of women following addition of bevacizumab to epirubicin and docetaxel treatment but not following treatment with epirubicin and docetaxel only.

Expression of SOX17 at the messenger RNA level from the tumors of patients enrolled in the PROMIX trial is graphically depicted here in primary tumors at baseline (left), in primary tumors following administration of 2 cycles of chemotherapy only (epirubicin and docetaxel) ("Chemo", middle), and in primary tumors at surgery, following addition of bevacizumab to treatment regimen ("Chemo + Bev", right). with mean SOX17 mRNA level marked and the result of a statistical test evaluating significance of difference in SOX17 expression between primary tumors of the breast and metastases to the brain in patients with breast cancer, a p-value, listed above. $* * * *: p<0.0001$. ns: not significant. 


\section{SOX17}

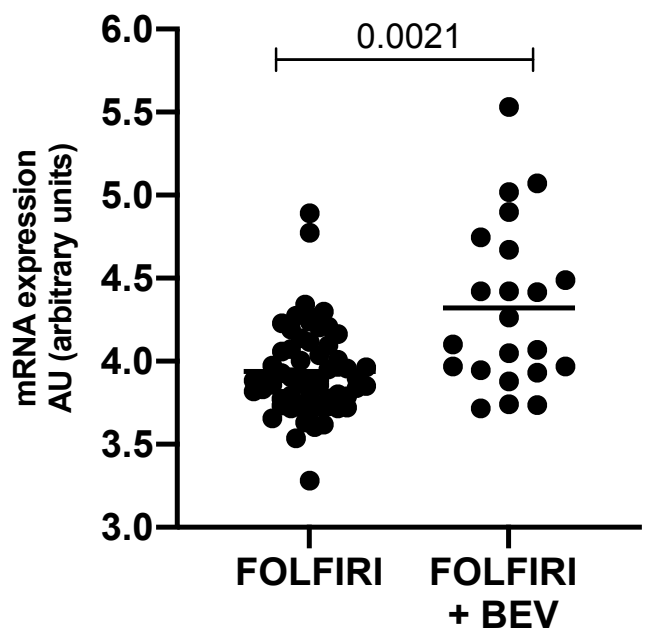

Figure 2: SOX17 is transcriptionally induced in the primary tumors of patients with colorectal cancer treated with bevacizumab and FOLFIRI.

Expression of SOX17 at the messenger RNA level from the tumors of patients with colorectal cancer treated with FOLFIRI (left), and in primary tumors of patients with colorectal cancer treated with FOLFIRI and bevacizumab (right), with mean SOX17 mRNA level marked and the result of a statistical test evaluating significance of difference in SOX17 expression between the primary tumors of patients with colorectal cancer treated with FOLFIRI or FOLFIRI and bevacizumab, a p-value, listed above.

PAGE 8 